\title{
ROOT SYSTEM OF A PERTURBATION OF A SELFADJOINT OPERATOR WITH DISCRETE SPECTRUM
}

\author{
JAMES ADDUCI AND BORIS MITYAGIN
}

\begin{abstract}
We analyze the perturbations $T+B$ of a selfadjoint operator $T$ in a Hilbert space $H$ with discrete spectrum $\left\{t_{k}\right\}, T \phi_{k}=t_{k} \phi_{k}$, as an extension of our constructions in [1] where $T$ was a harmonic oscillator operator. In particular, if $t_{k+1}-t_{k} \geq c k^{\alpha-1}, \quad \alpha>1 / 2$ and $\left\|B \phi_{k}\right\|=o\left(k^{\alpha-1}\right)$ then the system of root vectors of $T+B$, eventually eigenvectors of geometric multiplicity 1 , is an unconditional basis in $H$.
\end{abstract}

\section{Statement of main Results}

Let $H$ be a separable Hilbert space. Consider an operator $T$ with domain $\operatorname{dom} T$ whose spectrum consists of a countable set of eigenvalues $\tau=\left\{t_{k}\right\}_{k=1}^{\infty}$ with corresponding eigenvectors $\left\{\phi_{k}\right\}$,

$$
T \phi_{k}=t_{k} \phi_{k}
$$

which form an orthonormal basis in $H$. Let us also assume that $t_{k+1}-t_{k}>0$ and that for some fixed $p \in \mathbb{Z}_{+}, d>0$

$$
t_{k+p}-t_{k}>d \quad \forall k \in \mathbb{Z}_{+} .
$$

Define $\triangle t_{k}=t_{k+1}-t_{k}$. Then (1.1) says $\triangle t_{k}+\triangle t_{k+1}+\ldots+\triangle t_{k+p-1}>d \quad \forall k$. Hence, for any $k \in \mathbb{Z}_{+}$, there exists $\gamma(k) \in\{0,1, \ldots, p-1\}$ such that

(1) $\triangle t_{k+\gamma(k)} \geq d / p$ and

(2) $\triangle t_{k+j}<d / p \quad \forall j<\gamma(k)$.

Let $j_{1}=1$ and $j_{k}=j_{k-1}+\gamma\left(j_{k-1}\right)$ for $k>1$ and define $T_{k}=t_{j_{k}}$. Define the intervals

$$
F_{1}=\left[T_{1}-\frac{d}{2 p}, T_{2}+\frac{d}{2 p}\right], \quad F_{k}=\left[T_{k}+\frac{d}{2 p}, T_{k+1}+\frac{d}{2 p}\right], \quad k>1 .
$$

It follows that

$$
\tau \subset \cup_{k=1}^{\infty} F_{k} \quad \text { and } \quad \#\left(\tau \cap F_{k}\right) \leq p \quad \forall k
$$

Set

$$
\Pi_{k}=\left\{a+i b: a \in F_{k},|b| \leq \frac{d}{2 p}\right\}, \quad \Gamma_{k}=\partial \Pi_{k}
$$

and for $z \notin \mathrm{Sp} T$,

$$
R^{0}(z)=(z-T)^{-1}
$$

2000 Mathematics Subject Classification. 47E05, 34L40, 34L10. 
With

$$
P_{k}^{0}=\frac{1}{2 \pi i} \int_{\Gamma_{k}} R^{0}(z) d z
$$

we have a resolution of the identity $\sum_{k=1}^{\infty} P_{k}^{0}$.

Consider the perturbed operator $L=T+B$ with $B$ closed and $\operatorname{dom} B \supseteq$ $\operatorname{dom} T$. Set $\beta=\left\{\beta_{k}=\left\|B \phi_{k}\right\|^{2}\right\}$. In Proposition 1 we will use the condition

$$
\lim \sup \beta_{k}<\left(\frac{d}{2 p}\right)^{2}\left(\frac{1}{8 p\left(1+\pi^{2} / 3\right)}\right) .
$$

This condition implies the existence of integers $M, N$ such that

$$
\begin{aligned}
\beta_{k} & \leq\left(\frac{d}{2 p}\right)^{2}\left(\frac{1}{8 p\left(1+\pi^{2} / 3\right)}\right) \forall k \geq M, \\
\|\beta\|_{\infty} & \leq \frac{d^{2}}{16 p^{2}}\left(2 p \sum_{j=N+1}^{\infty} 1 / j^{2}\right)^{-1} .
\end{aligned}
$$

Define $h$ to be a positive constant which satisfies

$$
\sum_{j=0}^{\infty} \frac{1}{h^{2}+\left(\frac{j d}{2 p}\right)^{2}} \leq \frac{1}{8 p\|\beta\|_{\infty}}
$$

and set

$$
\begin{aligned}
\Pi_{0} & =\left\{a+i b:-h \leq a \leq T_{M+N+1}+\frac{d}{2 p},|b| \leq h\right\}, \quad \Gamma_{0}=\partial \Pi_{0}, \\
R(z) & =(z-L)^{-1} \quad \forall z \notin \operatorname{Sp} L .
\end{aligned}
$$

Proposition 1. Suppose the conditions (1.1) and (1.4) hold and that $M, N$ satisfy (1.5-1.6); $K=M+N$. Then, with the notation (1.2) - (1.3), SpL is discrete and contained in $\Pi_{0} \cup \cup_{j=K+1}^{\infty} \Pi_{j}$.

This proposition implies that the following operators are well-defined

$$
\begin{aligned}
S_{K} & =\frac{1}{2 \pi i} \int_{\partial \Gamma_{0}} R(z) d z \\
P_{k} & =\frac{1}{2 \pi i} \int_{\partial \Gamma_{k}} R(z) d z \quad \text { for } k \geq K+1 .
\end{aligned}
$$

Proposition 2. Under the conditions of Proposition 1,

$$
\begin{aligned}
\operatorname{dim} S_{K} & =\sum_{j=1}^{K} \operatorname{dim} P_{j}^{0} \leq p K, \\
\operatorname{dim} P_{j} & =\operatorname{dim} P_{j}^{0} \leq p \quad \text { for all } j \geq K+1 \quad \text { and } \\
\|R(z)\|^{2} & \leq\left(\frac{d}{p}\right)^{2} \quad \forall z \notin \Pi_{0} \cup \cup_{j=K+1}^{\infty} \Pi_{j} .
\end{aligned}
$$


Theorem 3. Suppose the condition (1.1) holds and $\left\|B \phi_{k}\right\| \rightarrow 0$ as $k \rightarrow \infty$. Then there is a bounded operator $W$ such that $W P_{k} W^{-1}=P_{k}^{0}, \operatorname{dim} P_{k}^{0} \leq p$ for all $k>K$ and $W S_{K} W^{-1}=\sum_{k=1}^{K} P_{k}^{0}$. Hence, $\left\{S_{K}, P_{K+1}, P_{K+2}, \ldots\right\}$ is a Riesz system of projections.

Basically this statement is proven in [9, Thm. 2] where the condition (1.4) is weaker (see (1.2) there) but the dimension of the projectors $\left\{P_{k}\right\}$ in the Riesz system are bounded by $2 p$, not by $p$. Our alternative approach -as in [1] - is based on the boundedness of the discrete Hilbert transform and its adjustments.

We will also consider the case in which the sequence of eigenvalues satisfies the growth condition

$$
t_{k+1}-t_{k} \geq \kappa k^{\alpha-1} \quad \forall k \in \mathbb{N}
$$

where $\alpha \in(0, \infty) \backslash\{1\}$.

Define

$$
v=2^{\frac{1}{\alpha-1 \mid}}
$$

and put

$$
V_{0}=[0, v) \cap \mathbb{N}, \quad V_{k}=\left[v^{k}, v^{k+1}\right) \cap \mathbb{N} \quad \forall k \in \mathbb{N} .
$$

Consider a closed operator $B$ with $\operatorname{dom} B \supseteq \operatorname{dom} T$ and

$$
\left\|B \phi_{k}\right\|=c_{k} k^{\alpha-1} \quad \text { with } \quad \lim _{k \rightarrow \infty} c_{k}=0 .
$$

(See the remark in Section 7.1). Set $L=T+B$ and $c_{\infty}=\sup \left|c_{k}\right|$.

For each $k \in \mathbb{N}$ define

$\Pi_{k}=\left\{a+i b: t_{k}-(\kappa / 2)(k-1)^{\alpha-1} \leq a \leq t_{k}+(\kappa / 2) k^{\alpha-1}, \quad|b| \leq(\kappa / 2) k^{\alpha-1}\right\}$

and $\Lambda_{k}=\partial \Pi_{k}$ so that

$$
\begin{aligned}
& \left|\Lambda_{k}\right| \leq 4 \kappa k^{\alpha-1} \quad \text { and } \\
& \left\{t_{k}\right\} \subset \cup_{1}^{\infty} \Pi_{j}
\end{aligned}
$$

Now select $N$ large enough so that

$$
\begin{aligned}
v^{N \alpha} & >c_{\infty}^{2}\left(\frac{1}{1-\frac{1}{v}}\right) \quad \text { and } \\
c_{j}^{2} & \leq(1 / 4)\left(\frac{16}{\kappa^{2}}\left(1+\frac{2 \pi^{2}}{3}\right)+\frac{4}{1-\frac{1}{v}}\right)^{-1} \quad \forall j \in V_{j}, \quad j \geq N / 2 .
\end{aligned}
$$


Finally set

$$
\begin{aligned}
\ell & =\sup \left\{\cup_{j \leq N} V_{j}\right\} \\
Y & =\left(4 c_{\infty}^{2} v \sum_{j=1}^{N}(\sqrt{v} / 2)^{2 j}\right)^{1 / 2} \text { and } \\
\Pi_{0} & =\left\{a+i b:-Y \leq a \leq t_{\ell}+(\kappa / 2) \ell^{\alpha-1}, \quad|b| \leq Y\right\}
\end{aligned}
$$

and define

$$
R^{0}(z)=(z-T)^{-1}, \quad R(z)=(z-L)^{-1},
$$

$$
Q_{j}^{0}=\frac{1}{2 \pi i} \int_{\Lambda_{j}} R^{0}(z) d z \quad \forall j \in \mathbb{N}, \quad Q_{j}=\frac{1}{2 \pi i} \int_{\Lambda_{j}} R(z) d z \quad \forall j>\ell \quad \text { and }
$$

$$
U_{\ell}=\frac{1}{2 \pi i} \int_{\Lambda_{0}} R(z) d z
$$

Proposition 4. Suppose the conditions (1.10) and (1.12) hold with $\alpha \in$ $(0, \infty) \backslash\{1\}$. Then SpL is discrete and eventually simple. Furthermore, with the notation (1.17)-(1.20), we have:

$$
\begin{aligned}
S p L & \subset \Pi_{0} \cup\left(\cup_{j=\ell+1}^{\infty} \Pi_{j}\right), \\
\operatorname{dim} U_{\ell} & =\sum_{j=1}^{\ell} \operatorname{dim} Q_{j}^{0}, \quad \text { and } \quad \operatorname{dim} Q_{j}=\operatorname{dim} Q_{j}^{0}=1 \quad \forall j>\ell .
\end{aligned}
$$

Proposition 5. Fix $n \in \mathbb{N}$ with $n>\ell$. Then for each $z \in \Lambda_{n}$ we have:

$$
\|R(z)\| \leq\left\{\begin{array}{l}
\kappa n^{1-\alpha} \quad \text { if } 1 / 2<\alpha<1, \\
\kappa(n-1)^{1-\alpha} \quad \text { if } 1<\alpha<\infty .
\end{array}\right.
$$

Theorem 6. Let $\alpha \in(1 / 2, \infty) \backslash\{1\}$ and suppose the condition (1.10) and (1.12) hold. Then there is a bounded operator $W$ such that $W U_{\ell} W^{-1}=$ $\sum_{k=1}^{\ell} Q_{k}^{0}$ and $W Q_{k} W^{-1}=Q_{k}^{0}$ for all $k>\ell$. Hence, $\left\{U_{\ell}, Q_{\ell+1}, Q_{\ell+2}, \ldots\right\}$ is a Riesz system of projections.

Let us notice that Propositions 4, 5 and Theorem 6 can be reformulated in an proper way for $\alpha=1$. This would necessitate additional notation. We refer the reader to our previous paper [1] where the case $\alpha=1$ is formulated and proven in detail.

\section{TECHNICAL PRELIMINARIES}

Define $B\left(\ell^{2}(\mathbb{N})\right)$ to be the space of all bounded linear operators on $\ell^{2}(\mathbb{N})$. Given a strictly increasing sequence of real numbers $a=\left(a_{k}\right)$ define the 
generalized discrete Hilbert transform (GDHT) by

$$
\left(G_{a} \xi\right)(n)=\sum_{k \neq n} \frac{\xi_{k}}{a_{k}-a_{n}}, \quad \xi=\left(\xi_{k}\right)_{k=1}^{\infty}
$$

Of course care must be taken to ensure that the right hand side of (2.1) is defined. If $a_{k}=k \quad \forall k$ we have the standard discrete Hilbert transform $(\mathrm{DHT}) G \in B\left(\ell^{2}(\mathbb{N})\right)$ (see HLP).

Lemma 7. Suppose $a_{k+1}-a_{k}>0$ and $a_{k} \in \mathbb{N} \forall k \in \mathbb{N}$. Then $G_{a} \in$ $B\left(\ell^{2}(\mathbb{N})\right)$.

Proof. Suppose $\xi \in \ell^{2}:=\ell^{2}(\mathbb{N})$. Define the operator $I_{a}$ by $I_{a}\left(e_{a_{k}}\right)=e_{a_{k}} \quad \forall k$ and $I_{a}\left(e_{j}\right)=0 \quad \forall j \notin a$ and define a vector $\tilde{\xi}$ by $\tilde{\xi}_{a_{k}}=\xi_{k} \quad \forall k$ and $\tilde{\xi}_{j}=$ $0 \forall j \notin a$. Then $\|\xi\|=\|\tilde{\xi}\|$ and $G_{a} \xi=I_{a} G I_{a} \tilde{\xi}$. Because $I_{a}$ and $G$ are bounded, $G_{a}$ is bounded as well.

Lemma 8. Suppose $A$ is an operator whose matrix entries satisfy

$$
\left|A_{k, j}\right| \leq \frac{C}{|k-j|^{2}}, \quad A_{k, k}=0 .
$$

Then $A \in B\left(\ell^{2}(\mathbb{N})\right)$ with $\|A\| \leq C \pi^{2} / 3$.

Proof. The proof is elementary (see for example Lemma 4 in [1]).

Lemma 9. Suppose

$$
a_{k+1}-a_{k}>\delta \quad \forall k
$$

Then $G_{a} \in B\left(\ell^{2}(\mathbb{N})\right)$ with $\left\|G_{a}\right\| \leq \frac{1}{\delta}\left(\frac{2 \pi^{2}}{3}+2\|G\|\right)$.

Proof. Write $\mathbb{R}=\cup_{k \in \mathbb{Z}} I_{k}, \quad I_{k}=[(k-1 / 2) \delta / 2,(k+1 / 2) \delta / 2)$. Then by (2.2), \# $\left(I_{k} \cap a\right)=0$ or 1 . Enumerate $\left\{j\left(\frac{\delta}{2}\right): \#\left(I_{j} \cap a\right)=1\right\}$ in increasing order and call the sequence $\tilde{a}$. It follows from Lemma 7 that the GDHT $G_{\tilde{a}} \in B\left(\ell^{2}(\mathbb{N})\right)$ with $\left\|G_{\tilde{a}}\right\| \leq(2 / \delta)\|G\|$. Thus, with $A:=G_{a}-G_{\tilde{a}}$ it suffices to show $\|A\| \leq \frac{2 \pi^{2}}{3 \delta}$.

Consider the matrix entries $A_{k, k}=0 \quad \forall k$, and for $j \neq k$,

$$
\left|A_{j, k}\right|=\left|\frac{1}{a_{j}-a_{k}}-\frac{1}{\tilde{a_{j}}-\tilde{a_{k}}}\right|=\left|\frac{\left(\tilde{a_{j}}-a_{j}\right)-\left(\tilde{a_{k}}-a_{k}\right)}{\left(a_{j}-a_{k}\right)\left(\tilde{a_{j}}-\tilde{a_{k}}\right)}\right| .
$$

By (2.2) we have $\left|a_{j}-a_{k}\right|>|j-k| \delta,\left|\tilde{a_{j}}-\tilde{a_{k}}\right| \geq|j-k|(\delta / 2)$, and $\mid \tilde{a_{j}}-$ $a_{j}|,| \tilde{a_{k}}-a_{k} \mid<\delta / 2$. Hence,

$$
\left|A_{j, k}\right| \leq \frac{\delta / 2+\delta / 2}{(|j-k|(\delta / 2))(|j-k| \delta)}=(2 / \delta) \frac{1}{|j-k|^{2}} .
$$

So by Lemma $8,\|A\| \leq \frac{2 \pi^{2}}{3 \delta}$. 
Lemma 10. Suppose $a_{k}$ is a strictly increasing sequence with $a_{k} \uparrow \infty$ and $\left(z_{k}\right)$ is a complex sequence satisfying

$$
\begin{aligned}
& \left|I m z_{k}\right|<\delta \\
& \operatorname{Re} z_{k} \in\left(a_{k-1}+\delta, a_{k+1}-\delta\right) \quad \text { and } \\
& \left|\operatorname{Re} z_{k}-a_{k}\right|<\Delta \quad \forall k .
\end{aligned}
$$

Then the operator $Z_{a}$ defined by

$$
\left(Z_{a} \xi\right)(n)=\sum_{k \neq n} \frac{\xi_{k}}{a_{k}-z_{n}}
$$

is bounded in $\ell^{2}$ with

$$
\left\|Z_{a}\right\| \leq \frac{1}{2 \delta}\|G\|+\frac{2 \delta \pi^{2}}{3 \Delta^{2}}
$$

Proof. By (2.3),$a_{k+1}-a_{k}>2 \delta \quad \forall k$. Hence, by Lemma 9, $\left\|G_{a}\right\| \leq \frac{1}{2 \delta}\|G\|$. Now, set $A=G_{a}-Z_{a}$. It suffices to show $\|A\| \leq \frac{2 \delta \pi^{2}}{3 \Delta^{2}}$. Consider the matrix elements $A_{k, k}=0 \quad \forall k$ and for $j \neq k$,

$$
\begin{aligned}
\left|A_{j, k}\right| & =\left|\frac{1}{a_{k}-a_{n}}-\frac{1}{a_{k}-z_{n}}\right|=\left|\frac{a_{n}-z_{n}}{\left(a_{k}-a_{n}\right)\left(a_{k}-z_{n}\right)}\right| \\
& \leq \frac{2 \delta}{\Delta|k-n| \Delta|k-n|}=\frac{2 \delta}{\Delta^{2}|k-n|^{2}} .
\end{aligned}
$$

It follows from Lemma 8 that $\|A\| \leq \frac{2 \delta \pi^{2}}{3 \Delta^{2}}$.

Define $\ell^{2}(H)$ with the norm

$$
\|\xi\|_{\ell^{2}(H)}^{2}=\sum_{j=1}^{\infty}\left\|\xi_{j}\right\|_{H}^{2}, \quad \xi=\left(\xi_{k}\right), \xi_{k} \in H
$$

Lemma 11. Supppose $a, z$, and $Z_{a}$ are as in Lemma [10. Consider the operator $Z_{a}^{V}$ in $\ell^{2}(H)$

$$
\left(Z_{a}^{V} \xi\right)(n)=\sum_{k \neq n} \frac{\xi_{k}}{a_{k}-z_{n}}
$$

Then $\left\|Z_{a}^{V}\right\|_{\ell^{2}(H)}=\left\|Z_{a}\right\|_{\ell^{2}} \leq \frac{1}{2 \delta}\|G\|+\frac{2 \delta \pi^{2}}{3 \Delta^{2}}$. 
Proof. Suppose $\xi=\left(\xi_{k}\right) \in \ell^{2}(H)$ with $\xi_{j}=\sum_{k=1}^{\infty} \xi_{j}^{(k)} \phi_{k} \in H$.

Then

$$
\begin{aligned}
\left\|Z_{a}^{V} \xi\right\|^{2} & =\sum_{n=1}^{\infty}\left\|\left(Z_{a}^{V} \xi\right)(n)\right\|^{2} \\
& =\sum_{n=1}^{\infty} \sum_{k=1}^{\infty}\left|\sum_{j \neq n} \frac{\xi_{j}^{(k)}}{a_{j}-z_{n}}\right|^{2}=\sum_{k=1}^{\infty} \sum_{n=1}^{\infty}\left|\left(Z_{a} \xi^{(k)}\right)(n)\right|^{2} \\
& =\sum_{k=1}^{\infty}\left\|Z_{a} \xi^{(k)}\right\|_{\ell^{2}}^{2} \leq\left\|Z_{a}\right\|^{2} \sum_{k=1}^{\infty}\left\|\xi^{(k)}\right\|_{H}^{2} \\
& =\left\|Z_{a}\right\|^{2}\|\xi\|_{\ell^{2}(H)}^{2} .
\end{aligned}
$$

We now move to a series of lemmas which will be used in the proofs of Proposition 4 and Theorem 6. The proofs of these lemmas for values of $0<\alpha<1$, and $\alpha>1$ follow a similar pattern so we only present proofs for values of $\alpha<1$.

Lemma 12. Suppose $\alpha>0, \alpha \neq 1,\left\{t_{k}\right\}_{1}^{\infty}$ satisfies (1.10), $m \in V_{M}$ and $n-1 \in V_{N}$ with $M \leq N-2$. Then $t_{n}-t_{m} \geq c(1-1 / v) v^{\alpha N}$ with $v \in$ (1.11).

Proof. We have

$$
\begin{aligned}
t_{n}-t_{m} & =\left(t_{n}-t_{n-1}\right)+\left(t_{n-1}-t_{n-2}\right)+\ldots+\left(t_{m+1}-t_{m}\right) \\
& \geq c\left[(n-1)^{\alpha-1}+(n-2)^{\alpha-1}+\ldots+m^{\alpha-1}\right] .
\end{aligned}
$$

Suppose first $\alpha \in(0,1)$. By the mean value theorem if $a<b$ we have $\alpha a^{\alpha-1} \geq b^{\alpha}-a^{\alpha} \geq \alpha b^{\alpha-1}$.

Hence,

$$
\begin{aligned}
c\left[(n-1)^{\alpha-1}\right. & \left.+(n-2)^{\alpha-1}+\ldots+m^{\alpha-1}\right] \geq(c / \alpha)\left[n^{\alpha}-m^{\alpha}\right] \\
& \geq(c / \alpha)\left[v^{N \alpha}-v^{(N-1) \alpha}\right] \geq(c / \alpha) \alpha v^{N(\alpha-1)}\left[v^{N}-v^{N-1}\right] \\
& =c(1-1 / v) v^{\alpha N} .
\end{aligned}
$$

A similar argument can be used for $\alpha>1$. We omit the details.

The following lemma generalizes the boundedness of the discrete Hilbert transform. It is a basic tool in our proof of Theorem 6. In fact, our proof of Theorem 6 only works for values of $\alpha>1 / 2$ because the following lemma does not hold for $\alpha \leq 1 / 2$ (see Remark 14).

Lemma 13. Suppose $\alpha \in(1 / 2, \infty)$ and $\left\{t_{k}\right\}_{1}^{\infty}$ satisfies (1.10). Then there is a constant $\tilde{C}>0$ depending only on $\alpha$ such that for any $\left(b_{m}\right) \in \ell^{2}(\mathbb{N})$ we 
have

$$
\sum_{n=1}^{\infty}\left|\sum_{m \neq n} \frac{m^{\alpha-1} b_{m}}{t_{m}-t_{n}}\right|^{2} \leq \tilde{C}\|b\|^{2} .
$$

Remark 14. Of course, (2.5) does not hold if $\sum 1 / t_{n}^{2}=\infty$ (even for $b=e_{1}$, i.e., $b(1)=1, \quad b(m)=0, m>1)$ so $t_{n}=n^{a}, \quad 0<a \leq 1 / 2$ or sequences with the growth condition (1.10) with $a \leq 1 / 2$ could not be analyzed with some analog of Lemma 13 or Lemma 15.

Proof. Let $b \in \ell^{2}(\mathbb{N})$. First suppose $1 / 2<\alpha<1$ so that $v$ from (1.11) can be written as $v=2^{2(1+\delta)}$ with $\delta>0$. Set $\gamma=\frac{1+2^{2 \delta}}{2}$. By Cauchy's inequality we have

$$
\begin{aligned}
& \sum_{n=1}^{\infty}\left|\sum_{m \neq n} \frac{m^{\alpha-1} b_{m}}{t_{m}-t_{n}}\right|^{2}=\sum_{N=1}^{\infty} \sum_{n \in V_{N}}\left|\sum_{M=1}^{\infty} \sum_{m \in V_{M}} \frac{m^{\alpha-1} b_{m}}{t_{m}-t_{n}}\right|^{2} \\
& \quad \leq\left(\frac{2}{1-\gamma^{-1 / 2}}\right) \sum_{N=1}^{\infty} \sum_{n \in V_{N}} \sum_{M=1}^{\infty} \gamma^{|N-M|}\left|\sum_{m \in V_{M}} \frac{m^{\alpha-1} b_{m}}{t_{m}-t_{n}}\right|^{2} \\
& \quad=\left(\frac{2}{1-\gamma^{-1 / 2}}\right)\left(S_{1}+S_{2}\right)
\end{aligned}
$$

where

$$
\begin{aligned}
& S_{1}=\sum_{N=1}^{\infty} \sum_{n \in V_{N}} \sum_{\substack{M=1 \\
|M-N|>1}}^{\infty} \gamma^{|N-M|}\left|\sum_{m \in V_{M}} \frac{m^{\alpha-1} b_{m}}{t_{m}-t_{n}}\right|^{2} \\
& S_{2}=\sum_{N=1}^{\infty} \sum_{n \in V_{N}} \sum_{\substack{M=1 \\
|M-N| \leq 1}}^{\infty} \gamma^{|N-M|}\left|\sum_{m \in V_{M}} \frac{m^{\alpha-1} b_{m}}{t_{m}-t_{n}}\right|^{2} .
\end{aligned}
$$

By Lemma 12 and another application of Cauchy's inequality

$$
\begin{aligned}
S_{1} & \leq\left[\sum_{N=1}^{\infty} \sum_{n \in V_{N}} \sum_{\substack{M=1 \\
|M-N|>1}}^{\infty} \gamma^{|N-M|} \sum_{m \in V_{M}} \frac{m^{2(\alpha-1)}}{\left(t_{m}-t_{n}\right)^{2}}\right] \sum_{m \in V_{M}} b_{m}^{2} \\
& \leq \sum_{M=1}^{\infty} \sum_{m \in V_{M}} b_{m}^{2} \sum_{\substack{N=1 \\
|N-M|>1}}^{\infty} \frac{\gamma^{|N-M|}\left(\# V_{M}\right) \cdot\left(\# V_{N}\right) 2^{-2 M}}{(v / 2)^{2 \max (M, N)}} \\
& \leq \sum_{M=1}^{\infty} \sum_{m \in V_{M}} b_{m}^{2} \sum_{|N-M|>1}^{\infty} \gamma^{|N-M|} v^{M+N+2} 2^{-2 M}(2 / v)^{2 \max (M, N)}
\end{aligned}
$$


We will show that the following is uniformly bounded in $M$

$$
\sum_{N=1}^{\infty} \gamma^{|N-M|} v^{-|M-N|} 2^{2 \max (M, N)-2 M} \text {. }
$$

We have

$$
\begin{aligned}
& \sum_{N=1}^{\infty} \gamma^{|N-M|} v^{-|M-N|} 2^{2 \max (M, N)-2 M} \\
& \quad=\sum_{N=1}^{\infty} \gamma^{|N-M|} 2^{-2(1+\delta)|M-N|-2 M} 2^{2 \max (M, N)} \\
& \quad \leq \sum_{N=1}^{\infty} \gamma^{|N-M|} 2^{-2 \max (M, N)-2 \delta|M-N|} 2^{2 \max (M, N)} \\
& \quad=\sum_{N=1}^{\infty} \gamma^{|N-M|}\left(2^{-2 \delta}\right)^{|M-N|} \leq \frac{2}{1-\frac{\gamma}{2^{2 \delta}}} .
\end{aligned}
$$

Combining this bound with (2.7), we conclude

$$
S_{1} \leq\left(\frac{2}{1-\frac{\gamma}{2^{2 \delta}}}\right)\|b\|^{2}
$$

Now

$$
\begin{aligned}
S_{2} & \leq \sum_{N=1}^{\infty} \sum_{n \in V_{N}} \sum_{|M-N| \leq 1} \gamma^{|M-N|}\left(\sum_{m \in V_{M}} \frac{m^{2(\alpha-1)}}{\left(t_{m}-t_{n}\right)^{2}}\right)\left(\sum_{m \in V_{M}}\left|b_{m}\right|^{2}\right) \\
& \leq \sum_{N=1}^{\infty} \sum_{|M-N| \leq 1}\left(\# F_{M}\right) \cdot\left(\# F_{N}\right) \gamma^{|M-N|} 2^{-2 M}(v / 2)^{-2 \max (M, N)}\left(\sum_{m \in V_{M}}\left|b_{m}\right|^{2}\right) \\
& \leq \sum_{N=1}^{\infty} \sum_{|M-N| \leq 1} v^{M+N+2} \gamma^{|M-N|} v^{-2 \max (M, N)} 2^{-2 M+2 \max (M, N)}\left(\sum_{m \in V_{M}}\left|b_{m}\right|^{2}\right) \\
& \leq 16 v^{4} \sum_{N=1}^{\infty} \sum_{|M-N| \leq 1}\left(\sum_{m \in V_{M}}\left|b_{m}\right|^{2}\right) \leq 3 \cdot 16 \cdot v^{4} \gamma\|b\|^{2} .
\end{aligned}
$$

Combining these bounds with (2.6) and (2.8) we have

$$
\sum_{n=1}^{\infty}\left|\sum_{m \neq n} \frac{m^{\alpha-1} b_{m}}{t_{m}-t_{n}}\right|^{2} \leq\left(\frac{2}{1-\gamma^{-1 / 2}}\right)\left(\left(\frac{2}{1-\frac{\gamma}{2^{2 \delta}}}\right)+3 \cdot 16 v^{4} \gamma\right)\|b\|^{2} .
$$

So (2.5) is proved for $1 / 2<\alpha<1$.

The proof for $\alpha>1$ is similar. We omit the details.

The following lemma can be proven in the same manner as Lemma 13 . We omit the details. 
Lemma 15. Suppose $\alpha \in(1 / 2, \infty) \backslash\{1\}$ and $\left\{t_{k}\right\}_{1}^{\infty}$ satisfies (1.10). Let $\left\{z_{k}\right\}$ be a sequence such that $\left|z_{k}-t_{k}\right| \leq(c / 2) k^{\alpha-1} \forall k \in \mathbb{N}$. Then there is a constant $C>0$ depending only on $\alpha$ such that for any $\left(b_{m}\right) \in \ell^{2}(\mathbb{N})$ we have

$$
\sum_{n=1}^{\infty}\left|\sum_{m \neq n} \frac{m^{\alpha-1} b_{m}}{t_{m}-z_{n}}\right|^{2} \leq C\|b\|^{2}
$$

The following vector-valued version of Lemma 15 can be proven in the same manner as Lemma 11. We omit the details.

Lemma 16. Suppose $\alpha \in(1 / 2, \infty) \backslash\{1\}$ and $\left\{t_{k}\right\}_{1}^{\infty}$ satisfies (1.10). Let $\left\{z_{k}\right\}$ be a sequence such that $\left|z_{k}-t_{k}\right| \leq(c / 2) k^{\alpha-1} \forall k \in \mathbb{N}$. Then there is a constant $C>0$ depending only on $\alpha$ such that for any $\left(b_{m}\right) \in \ell^{2}(H)$ we have

$$
\sum_{n=1}^{\infty}\left|\sum_{m \neq n} \frac{m^{\alpha-1} b_{m}}{t_{m}-z_{n}}\right|^{2} \leq C\|b\|^{2} .
$$

\section{Proof of Proposition 1 and Proposition 2}

Proof. Let $z \notin \cup_{0}^{\infty} \Pi_{k}$. To show $z \notin \mathrm{Sp} L$ it suffices to show $\left\|B R^{0}(z)\right\| \leq 1 / 2$ since then $R(z)=R^{0}(z)\left(I-B R^{0}(z)\right)^{-1}$ is well defined. To this end let $f \in H$ with $\|f\|^{2}=1, \quad f=\sum f_{k} \phi_{k}$.

We have

$$
\begin{aligned}
\left\|B R^{0}(z) f\right\|^{2} & =\left\|B R^{0}(z) \sum f_{k} \phi_{k}\right\|^{2}=\left\|\sum \frac{f B \phi_{k}}{z-t_{k}}\right\|^{2} \\
& \leq\|f\|^{2} \sum \frac{\beta_{k}}{\left|z-t_{k}\right|^{2}}=\sum \frac{\beta_{k}}{\left|z-t_{k}\right|^{2}} .
\end{aligned}
$$

Consider first the case $\operatorname{Re} z \in F_{m}$, for some $m \geq M+N+1$. We have

$$
\begin{aligned}
\sum \frac{\beta_{k}}{\left|z-t_{k}\right|^{2}} & =\sum \frac{\beta_{k}}{\left(\operatorname{Re} z-t_{k}\right)^{2}+\operatorname{Im} z^{2}} \\
& \leq\left(\frac{2 p}{d}\right)^{2}\left(\sum_{j \in F_{m}} \beta_{j}+\left[\sum_{J=1}^{N}+\sum_{J=N+1}^{\infty}\right] \frac{1}{J^{2}} \sum_{j \in F_{m \pm J}} \beta_{j}\right) .
\end{aligned}
$$

Because $m \geq M+N+1, m \pm N \geq M$ whenever $J<N$. So, by (1.5) we have

$$
\sum_{j \in F_{m}} \beta_{j}+\sum_{J=1}^{N} \frac{1}{J^{2}} \sum_{j \in F_{m \pm J}} \beta_{j} \leq p\left(1+2 \sum_{J=1}^{N} \frac{1}{J^{2}}\right)\left(\frac{1}{8 p\left(1+\pi^{2} / 3\right)}\right)\left(\frac{d}{2 p}\right)^{2}
$$

and by (1.6) we have

$$
\sum_{J=N+1}^{\infty} \frac{1}{J^{2}} \sum_{j \in F_{m \pm J}} \beta_{j} \leq 2 p\left(\sum_{J=N+1}^{\infty} \frac{1}{J^{2}}\right)\left(\frac{d^{2}}{16 p^{2}}\right)\left(p \sum_{J=N+1}^{\infty} \frac{1}{J^{2}}\right) .
$$


Combining (3.1) with $3.2+3.3$ we conclude that

$$
\left\|B R^{0}(z) f\right\|^{2} \leq 1 / 4
$$

whenever $\operatorname{Re} z \in F_{m}$ for some $m \geq M+N+1$.

Now consider the case $\operatorname{Re} z<T_{M+N+1}+\frac{d}{2 p}$, i.e. $\operatorname{Re} z \notin F_{m} \quad \forall m \geq$ $M+N+1$. Then $z \notin \cup_{0}^{\infty} \Pi_{j}$ implies $\left|z-t_{k}\right|^{2} \geq h^{2}+\operatorname{Re}\left(|z|-t_{k}\right)^{2}$.

Thus

$$
\begin{aligned}
\sum_{k=1}^{\infty} \frac{\beta_{k}}{\left|z-t_{k}\right|^{2}} & \leq \sum_{k=1}^{\infty} \frac{\beta_{k}}{h^{2}+\left(|\operatorname{Re} z|-t_{k}\right)^{2}} \\
& =\sum_{J=1}^{\infty} \sum_{j \in F_{J}} \frac{\beta_{k}}{h^{2}+\left(|\operatorname{Re} z|-t_{k}\right)^{2}} \\
& \leq 2 \sum_{J=0}^{\infty} \frac{p\|\beta\|_{\infty}}{h^{2}+\left(\frac{J d}{2 p}\right)^{2}} \leq \frac{2 p\|\beta\|_{\infty}}{8 p\|\beta\|_{\infty}}=1 / 4
\end{aligned}
$$

So we have shown that $\left\|R^{0}(z) B\right\|^{2} \leq 1 / 4$ for all $z \notin \Pi_{0} \cup \cup_{k=K+1}^{\infty} \Pi_{k}$. Thus, $\operatorname{Sp} L \subset \Pi_{0} \cup \cup_{k=K+1}^{\infty} \Pi_{k}$. Also, $\|R(z)\|=\left\|R^{0}(z)\left(I-B R^{0}(z)\right)^{-1}\right\| \leq$ $\left\|R^{0}(z)\right\|(1 / 2) \leq d / p$ for all $z \notin \Pi_{0} \cup \cup_{k=K+1}^{\infty} \Pi_{k}$.

A standard argument (see [5]) shows that

$$
\text { Trace } \frac{1}{2 \pi i} \int_{\Gamma_{n}}(z-T-t B)^{-1} d z, \quad 0 \leq t \leq 1,
$$

is a continuous integer-valued scalar function so it is constant and (1.7-1.8) hold.

\section{Proof of Theorem 3}

We first reproduce Lemma 4.17(a) from [4]. See also [3].

Lemma 17. Let $\left\{Q_{k}^{0}\right\}_{j \in \mathbb{Z}_{+}}$be a complete family of orthogonal projections in a Hilbert space $X$ and let $\left\{Q_{k}\right\}_{j \in \mathbb{Z}_{+}}$be a family of (not necessarily orthogonal) projections such that $Q_{j} Q_{k}=\delta_{j, k} Q_{j}$. Assume that

$$
\begin{array}{r}
\operatorname{dim}\left(Q_{0}^{0}\right)=\operatorname{dim}\left(Q_{0}\right)=m<\infty \\
\sum_{j=1}^{\infty}\left\|Q_{j}^{0}\left(Q_{j}-Q_{j}^{0}\right) u\right\|^{2} \leq c_{0}\|u\|^{2}, \quad \text { for every } u \in X
\end{array}
$$

where $c_{0}$ is a constant smaller than 1 . Then there is a bounded operator $W: X \rightarrow X$ with bounded inverse such that $Q_{j}=W^{-1} Q_{j}^{0} W$ for $j \in \mathbb{Z}_{+}$.

We are now ready to prove Theorem 3 ,

Proof. By Lemma 17 it suffices to show $\exists N_{*} \in \mathbb{N}$ such that for all $f \in H$ with $\|f\|=1$,

$$
\sum_{n \geq N_{*}}\left\|P_{n}^{0}\left(P_{n}-P_{n}^{0}\right) f\right\|^{2} \leq 1 / 2
$$


Fix $n>K$ (with $K$ from Proposition 1) and $f=\sum f_{k} \phi_{k} \in H$ with $\|f\|=1$. Then

$$
P_{n}-P_{n}^{0}=\frac{1}{2 \pi i} \int_{\Gamma_{n}}\left(R(z)-R^{0}(z)\right) d z=\frac{1}{2 \pi i} \int_{\Gamma_{n}} R(z) B R^{0}(z) d z .
$$

So by Proposition 2, inequality (1.9),

$$
\begin{aligned}
\left\|\left(P_{n}-P_{n}^{0}\right) f\right\|^{2} & =\frac{1}{2 \pi}\left\|\int_{\Gamma_{n}} R(z) B R^{0}(z) f d z\right\|^{2} \\
& \leq \frac{1}{2 \pi}\left[\int_{\Gamma_{n}}\left\|R(z) B R^{0}(z) f\right\|^{2} \leq C\left[\int_{\Gamma_{n}}\left\|B R^{0}(z) f\right\|\right]^{2}\right. \\
& =C\left[\int_{\Gamma_{n}}\left\|\sum_{k=1}^{\infty} \frac{f_{k} B \phi_{k}}{z-t_{k}}\right\| d z\right]^{2}
\end{aligned}
$$

where $C=\frac{1}{2 \pi}(d / p)^{2}$.

For $n \geq K$ define $z_{n}^{*} \in \Gamma_{n}$ to be a point where the maximum of the sum

$$
\left\|\sum_{k=1}^{\infty} \frac{f_{k} B \phi_{k}}{z-t_{k}}\right\|
$$

is attained. Note that $\left(z_{n}^{*}\right)$ depends on $f$. Since $\left|\Gamma_{k}\right| \leq 3 C \quad \forall k \geq 1$, we have by (4.1)

$$
\begin{aligned}
\left\|\left(P_{n}-P_{n}^{0}\right) f\right\|^{2} & \leq C\left|\Gamma_{n}\right|^{2}\left\|\sum_{k=1}^{\infty} \frac{f_{k} B \phi_{k}}{z-t_{k}}\right\|^{2} \\
& \leq C(3 d)^{2}\left\|\sum_{k=1}^{\infty} \frac{f_{k} B \phi_{k}}{z-t_{k}}\right\|^{2} .
\end{aligned}
$$

Suppose, for now, that $p=1$ so that $\#\left(\tau \cap F_{k}\right) \leq 1 \forall k \geq 1$. We will show that given any $\epsilon>0$ if we choose $N_{1}$ as in (4.5) below, then

$$
\sum_{n \geq N_{1}}\left\|\sum_{k=1}^{\infty} \frac{f_{k} B \phi_{k}}{z_{n}^{*}-t_{k}}\right\|^{2}<\epsilon \quad \forall\|f\|=1 .
$$

Note that $z_{n}^{*} \in \Gamma_{n}$ depends on $f$.

Recall that $G$ is the cannonical discrete Hilbert transform and set

$$
C_{1}=4\left(\frac{d\|G\|}{p}+\frac{\pi^{2}}{3 d p}\right) .
$$

Select $M_{1}$ large enough so that

$$
\left\|B \phi_{k}\right\|^{2} \leq \epsilon / C_{1} \quad \forall k \geq M_{1}
$$

and $N_{1}$ large enough so that whenever $w \in \Gamma_{n} \quad \forall n \geq K$

$$
\sum_{n=N_{1}}^{\infty}\left|w-t_{M_{1}}\right|^{-2} \leq \frac{\epsilon}{4\|\beta\|_{\infty} M_{1}} \quad \forall m \leq M_{1} .
$$


Then

$$
\left\|\sum_{k=1}^{\infty} \frac{f_{k} B \phi_{k}}{z_{n}^{*}-t_{k}}\right\|^{2} \leq 2\left[\left\|\sum_{k \leq M_{1}} \frac{f_{k} B \phi_{k}}{z_{n}^{*}-t_{k}}\right\|^{2}+\left\|\sum_{k>M_{1}} \frac{f_{k} B \phi_{k}}{z_{n}^{*}-t_{k}}\right\|^{2}\right] .
$$

By Cauchy's inequality we have

$$
\left\|\sum_{k \leq M_{1}} \frac{f_{k} B \phi_{k}}{z_{n}^{*}-t_{k}}\right\|^{2} \leq\left(\sum_{k \leq M_{1}}\left|f_{k}\right|^{2}\left\|B \phi_{k}\right\|^{2}\right)\left(\sum_{k \leq M_{1}}\left|z_{n}^{*}-t_{k}\right|^{-2}\right) .
$$

So by (4.5)

$$
2 \sum_{n \geq N_{1}}\left\|\sum_{k \leq M_{1}} \frac{f_{k} B \phi_{k}}{z_{n}^{*}-t_{k}}\right\|^{2} \leq\|\beta\|_{\infty} M_{1} \sum_{n \geq N_{1}}\left|z_{n}^{*}-t_{M_{1}}\right|^{-2}<\epsilon / 2 .
$$

It follows from Lemma 11 and (4.4) that

$$
\begin{aligned}
2 \sum_{n \geq N_{1}}\left\|\sum_{k>M_{1}} \frac{f_{k} B \phi_{k}}{z_{n}^{*}-t_{k}}\right\|^{2} & \leq 2\left\|\left(f_{k} B \phi_{k}\right)_{k=M_{1}}^{\infty}\right\|_{\ell^{2}(H)}^{2} C_{1} \\
& \leq \sup _{k \geq M_{1}}\left\|B \phi_{k}\right\|^{2} C_{1}<\epsilon / 2 .
\end{aligned}
$$

Hence, combining (4.6) and (4.7) we have proven (4.3).

Now suppose $p>1$. Reindex the sequences $t_{k}, f_{k}$, and $\phi_{k}$ in such a way that for all $k \geq 1$

$$
\tau \cap F_{k}=\left\{t_{k}^{(1)} \leq t_{k}^{(2)} \leq \ldots \leq t_{k}^{\left(J_{k}\right)}\right\}, \quad J_{k} \leq p .
$$

Then for $\tilde{K} \geq K$,

$$
\begin{gathered}
C \sum_{n \geq \tilde{K}}\left\|\sum_{k=1}^{\infty} \frac{f_{k} B \phi_{k}}{z_{n}^{*}-t_{k}}\right\|^{2}=C \sum_{n \geq \tilde{K}}\left\|\sum_{k=1}^{\infty} \sum_{j=1}^{J_{k}} \frac{f_{k}^{(j)} B \phi_{k}^{(j)}}{z_{n}^{*}-t_{k}^{(j)}}\right\|^{2} \\
\leq 2^{p} C \sum_{j=1}^{p} \sum_{n \geq \tilde{K}}\left\|\sum_{k=1}^{\infty} \frac{f_{k}^{(j)} B \phi_{k}^{(j)}}{z_{n}^{*}-t_{k}^{(j)}}\right\|^{2} .
\end{gathered}
$$

Note that if $J_{k}<p$ some terms in the series are taken to be 0 . For each $j \leq p$ the sequence $t_{1}^{(j)} \leq t_{2}^{(j)} \leq \ldots$ satisfies (1.1) with $p=1$. So by taking $\epsilon=1 /\left(2 \cdot 2^{p} C\right)$ and applying (4.3) for each $j \leq p$ the Theorem is proven by (4.2).

\section{Proof of Proposition 4 and Proposition 5}

Suppose that $z \notin \Pi_{0} \cup \cup_{j=\ell+1}^{\infty} \Pi_{j}$. We will show that $\left\|R^{0}(z) B\right\|^{2} \leq 1 / 2$. It follows that

$$
R(z)=\left(I-R^{0}(z) B\right)^{-1} R^{0}(z)
$$


is well defined. Let $f \in H$ with $\|f\|=1, f=\sum f_{k} \phi_{k}$.

Then

$$
\begin{aligned}
\left\|R^{0}(z) B f\right\|^{2} & =\left\|\sum f_{j} B R^{0}(z) \phi_{j}\right\|^{2} \\
& =\left\|\sum f_{j} \frac{B \phi_{j}}{z-t_{j}}\right\|^{2} \leq\left[\sum\left|f_{j}\right| \frac{\left\|B \phi_{j}\right\|}{\left|z-t_{j}\right|}\right]^{2} \\
& \leq \sum \frac{\left\|B \phi_{j}\right\|^{2}}{\left|z-t_{j}\right|^{2}}=\sum \frac{c_{j}^{2} j^{2(\alpha-1)}}{\left|z-t_{j}\right|^{2}} .
\end{aligned}
$$

Suppose first that $\operatorname{Re} z>t_{\ell}+\left(c_{\ell} / 2\right) \ell^{\alpha-1}$ so that $\operatorname{Re} z \in\left[v^{\tilde{N}}, v^{\tilde{N}+1}\right)$ for some $\tilde{N}>N$. Then

$$
\sum_{j=1}^{\infty} \frac{c_{j}^{2} j^{2(\alpha-1)}}{\left|z-t_{j}\right|^{2}}=S_{1}+S_{2}
$$

with

$$
S_{1}=\sum_{J=1}^{\tilde{N}-1-N / 2} \sum_{j \in V_{J}} \frac{c_{j}^{2} j^{2(\alpha-1)}}{\left|z-t_{j}\right|^{2}}, \quad S_{2}=\sum_{J=\tilde{N}-N / 2}^{\infty} \sum_{j \in V_{J}} \frac{c_{j}^{2} j^{2(\alpha-1)}}{\left|z-t_{j}\right|^{2}} .
$$

We have

$$
S_{1} \leq c_{\infty}^{2} \sum_{J=1}^{\tilde{N}-1-N / 2} \sum_{j \in V_{J}} \frac{j^{2(\alpha-1)}}{\left|z-t_{j}\right|^{2}} .
$$

If $0<\alpha<1$, then for each $J \leq \tilde{N}-1-N / 2$,

$$
\begin{aligned}
\sum_{j \in V_{J}} \frac{j^{2(\alpha-1)}}{\left|z-t_{j}\right|^{2}} & \leq \# F_{J} \frac{2^{-2 J}}{v^{2(\tilde{N}-1) \alpha}} \\
& =\# F_{J} 2^{-2 J}(v / 2)^{2}(v / 2)^{-2 \tilde{N}} \leq v^{J+1} 2^{-2 J}(v / 2)^{2}(2 / v)^{2 \tilde{N}} \\
& =(2 / v)^{2(\tilde{N}-J)-2} v^{-J} v \leq v(2 / v)^{N} v^{-J} .
\end{aligned}
$$

It follows from (1.15) that

$$
S_{1} \leq c_{\infty}^{2} v(2 / v)^{N} \sum_{J=1}^{\tilde{N}-1-N / 2} v^{-J} \leq c_{\infty}^{2}(2 / v)^{N}\left(\frac{1}{1-1 / v}\right)<1 / 4 .
$$

If $\alpha>1$, then for each $J \leq \tilde{N}-1-N / 2$ we have

$$
\begin{aligned}
\sum_{j \in V_{J}} \frac{j^{2(\alpha-1)}}{\left|z-t_{j}\right|^{2}} & \leq \# V_{J} \frac{2^{2 J}}{v^{2(\tilde{N}-1) \alpha}} \leq v^{J+1} \frac{2^{2 J}}{(2 v)^{2(\tilde{N}-1)}} \\
& =v^{J-2 \tilde{N}+3} 2^{2 J-2 \tilde{N}+2} \leq v(2 v)^{2 J-2 \tilde{N}+2} v^{-J} \\
& \leq v(2 v)^{2(J-\tilde{N})+2} v^{-J} \leq v(2 v)^{-N} v^{-J} .
\end{aligned}
$$


So it follows from (1.15) that

$$
S_{1} \leq c_{\infty}^{2} v(2 v)^{-N} \sum_{J=1}^{\tilde{N}-1-N / 2} v^{-J} \leq c_{\infty}^{2}(2 v)^{-N}\left(\frac{1}{1-1 / v}\right)<1 / 4
$$

Now

$$
\begin{aligned}
S_{2} & =\sum_{J=\tilde{N}-N / 2}^{\infty} \sum_{j \in V_{J}} \frac{c_{j}^{2} j^{2(\alpha-1)}}{\left|z-t_{j}\right|^{2}} \\
& \leq \sup _{\substack{j \in V_{J} \\
J \geq \tilde{N}-N / 2}}\left[\left(\sum_{\substack{J=\tilde{N}-N / 2 \\
J \neq \tilde{N}-1, \tilde{N}, \tilde{N}+1}}^{\infty}+\sum_{J=\tilde{N}-1, \tilde{N}, \tilde{N}+1}\right) \sum_{j \in V_{J}} \frac{j^{2(\alpha-1)}}{\left|z-t_{j}\right|^{2}}\right] .
\end{aligned}
$$

Let $\operatorname{Re} z \in\left(t_{k}, t_{k+1}\right], k \in V_{\tilde{N}-1} \cup V_{\tilde{N}} \cup V_{\tilde{N}+1}$. Then

$$
\left|z-t_{k}\right|^{2} \geq(v / 2)^{2} k^{2(\alpha-1)} \geq(v / 2)^{2} 2^{2(\tilde{N}-1)}
$$

and for $j \neq k, j \in V_{\tilde{N}-1} \cup V_{\tilde{N}} \cup V_{\tilde{N}+1}$ we have

$$
\left|z-t_{j}\right|^{2} \geq(\kappa / 2)^{2}|j-k|^{2} 2^{2(\tilde{N}-1)} .
$$

Thus,

$$
\begin{aligned}
& \sum_{J=\tilde{N}-1, \tilde{N}, \tilde{N}+1} \sum_{j \in V_{J}} \frac{j^{2(\alpha-1)}}{\left|z-t_{j}\right|^{2}} \\
& \quad \leq \frac{2^{2(\tilde{N}+1)}}{(\kappa / 2)^{2} 2^{2(\tilde{N}-1)}}+\sum_{\substack { J=\tilde{N}-1, \tilde{N}, \tilde{N}+1 \\
\begin{subarray}{c}{j \in V_{J} \\
j \neq k{ J = \tilde { N } - 1 , \tilde { N } , \tilde { N } + 1 \\
\begin{subarray} { c } { j \in V _ { J } \\
j \neq k } }\end{subarray}} \frac{2^{2(\tilde{N}+1)}}{(v / 2)^{2}|j-k|^{2} 2^{2(\tilde{N}-1)}} \\
& \quad \leq \frac{16}{c^{2}}\left(1+2 \sum_{j=1}^{\infty} j^{-2}\right)=\frac{16}{\kappa^{2}}\left(1+2 \pi^{2} / 3\right) .
\end{aligned}
$$

Furthermore, for $0<\alpha<1$

$$
\begin{aligned}
\sum_{J=\tilde{N}-N / 2}^{\infty} \sum_{j \in V_{J}} \frac{j^{2(\alpha-1)}}{\left|z-t_{j}\right|^{2}} & \leq \sum_{\substack{J=\tilde{N}-N / 2 \\
J \neq \tilde{N}-1, \tilde{N}, \tilde{N}+1}}^{\infty} \frac{\left(\# F_{J}\right) 2^{-2 J}}{(v / 2)^{2(J-1)}} \\
& \leq 4 \sum_{J=\tilde{N}-M}^{\infty} \frac{v^{J+1}}{v^{2 J-2}} \leq 4 v \sum_{J=1}^{\infty} v^{-J} \leq 4\left(\frac{1}{1-1 / v}\right) .
\end{aligned}
$$

By a similar argument, if $\alpha>1$ we also have

$$
\sum_{J=\tilde{N}-N / 2}^{\infty} \sum_{j \in V_{J}} \frac{j^{2(\alpha-1)}}{\left|z-t_{j}\right|^{2}} \leq 4\left(\frac{1}{1-1 / v}\right) .
$$


Hence, by (1.16)

$$
S_{2} \leq \sup _{\substack{j \in V_{J} \\ J \geq N-N / 2}}\left(\left(16 / \kappa^{2}\right)\left(1+2 \pi^{2} / 3\right)+\frac{4}{1-1 / v}\right)<1 / 4 .
$$

Now suppose that $\operatorname{Re} z \leq t_{\ell}+(\kappa / 2) \ell^{\alpha-1}$ so that $\operatorname{dist}(z, \tau)^{2} \geq Y^{2}$. Suppose $0<\alpha<1$. Then

$$
\begin{gathered}
\sum_{j=1}^{\infty} \frac{c_{j}^{2} j^{2(\alpha-1)}}{\left|z-t_{j}\right|^{2}} \leq c_{\infty}^{2} \sum_{J=1}^{N} \frac{\left(\# V_{J}\right) 2^{-2 J}}{Y^{2}}+\sup _{\substack{j \in V_{J} \\
j \geq N}} c_{j}^{2} \sum_{J=N+1}^{\infty} \frac{\left(\# V_{J}\right) 2^{-2 J}}{v^{2 \alpha(J-1)}} \\
\leq \frac{c_{\infty}^{2} v}{Y^{2}} \sum_{J=1}^{N}(\sqrt{v} / 2)^{2 J}+\sup _{\substack{j \in V_{J} \\
J \geq N}} \sum_{J=N+1}^{\infty}(2 / v)^{2} v^{1-J}<1 / 2
\end{gathered}
$$

by (1.16) and (1.17). We have shown

$$
\left\|\left(I-R^{0}(z) B\right)^{-1}\right\| \leq 2
$$

so that by (5.1) $R(z)$ is well-defined.

By a similar argument for $\alpha>1$ we also have

$$
\sum_{j=1}^{\infty} \frac{c_{j}^{2} j^{2(\alpha-1)}}{\left|z-t_{j}\right|^{2}} \leq 1 / 2
$$

We omit the details.

Now, definition (1.13) implies that for $z \in \Lambda_{n}$,

$$
\left\|R^{0}(z)\right\| \leq\left\{\begin{array}{l}
(\kappa / 2) n^{1-\alpha} \quad \text { if } \quad 1 / 2<\alpha \leq 1, \\
(\kappa / 2)(n-1)^{1-\alpha} \quad \text { if } \quad 1<\alpha<\infty .
\end{array}\right.
$$

Hence, inequality (1.22) follows from (5.1) and (5.2) together with (1.14) and (5.3).

\section{Proof of Theorem 6}

Proof. For the case of $\alpha=1$, this theorem is proven in the paper [1]. Henceforth, we assume $\alpha \neq 1$. By Lemma 17 it suffices to show there exists an integer $N_{*}$ such that

$$
\sum_{n \geq N_{*}}\left\|Q_{n}^{0}\left(Q_{n}-Q_{n}^{0}\right) f\right\|^{2} \leq 1 / 2
$$

Fix $n>N$ and $f=\sum f_{k} \phi_{k} \in H$ with $\|f\|=1$. Then

$$
\begin{aligned}
Q_{n}-Q_{n}^{0} & =\frac{1}{2 \pi i} \int_{\Lambda_{n}}\left(R(z)-R^{0}(z)\right) d z \\
& =\frac{1}{2 \pi i} \int_{\Lambda_{n}} R(z) B R^{0}(z) d z
\end{aligned}
$$


Hence,

$$
\begin{aligned}
\left\|\left(Q_{n}-Q_{n}^{0}\right)\right\|^{2} & =\frac{1}{2 \pi}\left\|\int_{\Lambda_{n}} R(z) B R^{0}(z) f d z\right\|^{2} \\
& \leq \frac{1}{2 \pi}\left[\int_{\Lambda_{n}}\left\|R(z) B R^{0}(z) f\right\| d z\right]^{2} \\
& =\frac{1}{2 \pi}\left[\int_{\Lambda_{n}}\left\|\sum_{k=1}^{\infty} \frac{f_{k} R(z) B \phi_{k}}{z-t_{k}}\right\| d z\right]^{2} .
\end{aligned}
$$

Now define $z_{n}^{*} \in \Lambda_{n}$ to be a point at which the following sum attains its maximum,

$$
\left\|\sum_{k=1}^{\infty} \frac{f_{k} B \phi_{k}}{z-t_{k}}\right\| \quad z \in \Lambda_{n} .
$$

Combining (1.14) with (1.22) yields

$$
\left|\Lambda_{n}\right|^{2}\|R(z)\|^{2} \leq 16 \kappa^{2} .
$$

So,

$$
\left\|\left(Q_{n}-Q_{n}^{0}\right)\right\|^{2} \leq \frac{16 \kappa^{2}}{2 \pi}\left[\left\|\sum_{k=1}^{\infty} \frac{f_{k} B \phi_{k}}{z_{n}^{*}-t_{k}}\right\|\right]^{2} .
$$

Recall the constant $C$ from Lemma 16. Condition (1.12) implies that there exists an absolute constant $N_{*}$ such that

$$
\left\|B \phi_{k}\right\| \leq \frac{2 \pi}{32 \kappa^{2} C} k^{\alpha-1} \quad \forall k \geq N_{*} .
$$

Thus

$$
\begin{aligned}
\sum_{n \geq N_{*}}\left\|Q_{n}^{0}\left(Q_{n}-Q_{n}^{0}\right) f\right\|^{2} & \leq \sum_{n \geq N_{*}}\left\|\left(Q_{n}-Q_{n}^{0}\right) f\right\|^{2} \\
& \leq \frac{16 \kappa^{2}}{2 \pi} \sum_{n \geq N_{*}}\left[\left\|\sum_{k=1}^{\infty} \frac{f_{k} B \phi_{k}}{z_{n}^{*}-t_{k}}\right\|\right]^{2} .
\end{aligned}
$$

Finally, combining (6.3) with Lemma 16 and (6.2) yields (6.1) and the proof is complete.

\section{Further REMARKS}

7.1. Our statement of Theorem 6 required the condition

$$
\lim _{k \rightarrow \infty} c_{k}=0
$$

where $\left\{c_{k}\right\}$ is defined in (1.12). With a careful accounting of quantities appearing in the proof of Theorem [6] we could have written a constant $c^{*}$ such that the condition (17.1) could be replaced by the weaker condition

$$
\limsup c_{k} \leq c^{*} .
$$


However, condition (7.1) or (7.2) could not be weakened in a significant way: an assumption $\lim \sup c_{k}<\infty$ would not guarantee the statement of Theorem 6. A counterexample in the case $\alpha=1$ is given in [1, Section 6.3.

Now we'll adjust the constructions of [1] to get an operator $B$, with

$$
\sup _{m}\left\{\left\|B \phi_{k}\right\|\left(t_{2 m}-t_{2 m-1}\right)^{-1}, \quad k=2 m-1,2 m\right\}=1 / 2
$$

such that the perturbation $L=T+B$ has a discrete spectrum, all points of $\operatorname{Sp}(T+B)$ are simple eigenvalues, the system $\left\{\psi_{k}\right\}$ of eigenvectors of $L$ is complete, but it is not a basis in $H$. If $t_{n}=n^{\alpha}, \quad 0<\alpha<\infty$, then (7.3) guarantees that $c_{\infty} \leq 1 / 2$.

Special 2-dimensional blocks play an important role in this construction. Put

$$
b=\left[\begin{array}{rr}
0 & s \\
-s & 0
\end{array}\right], \quad 0<s<1, \quad s^{2}+h^{2}=1, \quad 0<h<<1 .
$$

This choice is a slight adjustment of a 2-dimensional block (64) in [1]. It simplifies elementary calculations of the $\operatorname{Angle}\left(g^{+}, g^{-}\right)$, etc., for example. Such a block (7.4) could be used to get the same counterexample in Section 6.3, [1] instead of (64) there. Of course, $\|b\|=s$ in $\mathbb{C}^{2}$ in the Euclidean norm.

We have

$$
\left[\begin{array}{ll}
0 & 0 \\
0 & 2
\end{array}\right]+b=\left[\begin{array}{rr}
1 & 0 \\
0 & -1
\end{array}\right]+c, \quad c=\left[\begin{array}{ll}
-1 & s \\
-s & 1
\end{array}\right]
$$

and

$$
\begin{aligned}
c g^{ \pm} & = \pm h g^{ \pm} \quad \text { where } \\
g^{ \pm} & =\left(1, G^{ \pm 1}\right), \quad G=\sqrt{\frac{1+h}{1-h}} .
\end{aligned}
$$

If $\alpha=\operatorname{Angle}\left(g^{+}, g^{-}\right)$then

$$
(\cos \alpha)^{2}=\frac{\left(g^{+}, g^{-}\right)^{2}}{\left\|g^{+}\right\|^{2} \cdot\left\|g^{-}\right\|^{2}}=1-h^{2}=s^{2} .
$$

So $\sin \alpha=h$.

If $f=\Phi_{0}(f) u_{0}+\Phi_{1}(f) u_{1}$ is the standard basis decomposition in $\mathbb{C}^{2}$ then

$$
\left\|\Phi_{0}\right\|=\left\|\Phi_{1}\right\|=1 / \sin \alpha=1 / h .
$$

Now we define $B=\{b(m)\}$ where $b(m)$ are 2-dimensional blocks

$$
\frac{1}{2}\left(t_{2 m}-t_{2 m-1}\right)\left[\begin{array}{rr}
0 & s \\
-s & 0
\end{array}\right], \quad s=s(m), \quad \text { say } \quad s(m)^{2}+(1 / m)^{2}=1,
$$

on $\mathbb{C}^{2}=E_{m}:=\operatorname{Span}\left\{\phi_{2 m-1} ; \phi_{2 m}\right\}$. 
Then $E_{m}$ are invariant subspaces of $T+B$ and , (compare to [1], Lemma 14),

$$
(T+B)_{m}=\frac{1}{2}\left(t_{2 m}+t_{2 m-1}\right)+\frac{1}{2}\left(t_{2 m}-t_{2 m-1}\right)\left[\begin{array}{ll}
-1 & s \\
-s & 1
\end{array}\right]
$$

and

$$
(T+B)_{m} \psi_{m}^{ \pm}=\left(\frac{1}{2}\left(t_{2 m}+t_{2 m-1}\right) \pm \frac{1}{2}\left(t_{2 m}-t_{2 m-1}\right) h\right), \quad h=1 / m,
$$

where

$$
\psi_{m}^{ \pm}=g^{ \pm}(m)=\phi_{2 m-1}+G^{ \pm 1} \phi_{2 m} .
$$

We omit further details. With the explicit formulas given it is easy to see that (7.5) with $h=1 / m$ guarantees that $\left\{\psi_{m}^{ \pm}\right\}_{1}^{\infty}$ is not a basis.

7.2. As an application of Theorem 5, consider the differential operator $T$ on $L^{2}(\mathbb{R})$ defined by

$$
T y=-y^{\prime \prime}+|x|^{\beta} y, \quad \text { with } \quad \beta>1 .
$$

The spectrum of $T$ consists of an infinite set of eigenvalues

$$
\operatorname{Spec} T=\left\{\lambda_{0} \leq \lambda_{1} \leq \lambda_{2} \leq \ldots\right\} \quad \text { with } \quad \lim _{n \rightarrow \infty} \lambda_{n}=\infty .
$$

The growth of the sequence of eigenvalues is described by the formula

$$
\lim _{n \rightarrow \infty}\left[2 \int_{0}^{\lambda_{n}^{1 / \beta}}\left(\lambda_{n}-|x|^{\beta}\right)^{1 / 2} d x-(n+1 / 2) \pi\right]=0 .
$$

For a proof, see the last section of [10]. It follows from (7.7) by a change of variables that

$$
\lim _{n \rightarrow \infty}\left[2 \lambda_{n}^{\frac{2+\beta}{2 \beta}} \Omega_{\beta}-(n+1 / 2) \pi\right]=0 \quad \text { with } \quad \Omega_{\beta}=2 \int_{0}^{1}\left(1-x^{\beta}\right)^{1 / 2} d x
$$

Subtracting the $n^{\text {th }}$ term from the $n+1^{\text {st }}$ term in (17.8) we derive

$$
\lim _{n \rightarrow \infty}\left[\lambda_{n+1}^{\frac{2+\beta}{2 \beta}}-\lambda_{n}^{\frac{2+\beta}{2 \beta}}\right]=\pi / \Omega_{\beta} .
$$

From (7.9) it is straightforward to show that there exist constants $C>0$, $N \in \mathbb{N}$ (depending on $\beta$ ) such that

$$
\lambda_{n+1}-\lambda_{n} \geq C n^{\alpha-1} \quad \forall n>N, \quad \alpha=\frac{2 \beta}{\beta+2} .
$$

Let us mention the papers [7, [8] where the eigenvalues for the eigenproblem $-y_{z z}+q(z) y=\lambda y$ are analyzed for polynomial $q(z)$.

Denote the eigenfunction corresponding to $\lambda_{n}$ by $\phi_{n}$ and define

$$
L(p ; \alpha)=\left\{b: b(x)\left(1+|x|^{2}\right)^{-\alpha / 2} \in L^{p}(\mathbb{R})\right\} .
$$


We have the following bound on the behavior of $\phi_{n}$

$$
\begin{array}{r}
\left|\phi_{n}(x)\right| \leq \frac{K \exp (Q(x))}{\left.\left.\left|\lambda_{n}-\right| x\right|^{\beta}\right|^{1 / 4}+\lambda_{n}^{\frac{\beta-1}{6 \beta}}} \quad \text { with } \\
Q(x)= \begin{cases}-\int_{\lambda_{n}^{1 / \beta}}^{x}\left(\lambda_{n}-|t|^{\beta}\right)^{1 / 2} d t, & x>\lambda_{n}^{1 / \beta} \\
0, & |x| \leq \lambda_{n}^{1 / \beta} \\
\int_{\lambda_{n}^{1 / \beta}}^{x}\left(\lambda_{n}-|t|^{\beta}\right)^{1 / 2} d t, & x<-\lambda_{n}^{1 / \beta} .\end{cases}
\end{array}
$$

For the case $\beta=2$, this inequality is proven in [2, a few changes to this proof boost it to cover $\beta>1$. We omit the details. Such constructions for Schrodinger operators with turning points are discussed in [6, Ch 8,11].

By an argument like that given for Lemma 8 in [1] it follows from (7.11) that if $b \in L(p ; \alpha)$ then $\left\|b \phi_{n}\right\|_{2} \leq C n^{\frac{2 \beta \xi}{\beta+2}}$ where

$$
\begin{aligned}
\xi & =\max \left\{\frac{1}{3 \beta}(1-\beta+3 \alpha+(\beta-1) / p) ; \frac{1}{\beta}(\alpha-\beta / 4+1 / 2-1 / p)\right\} \\
& = \begin{cases}\frac{1}{3 \beta}(1-\beta+3 \alpha+(\beta-1) / p), & 2 \leq p<4 \\
\left.\frac{1}{\beta}(\alpha-\beta / 4+1 / 2-1 / p)\right\}, & 4<p .\end{cases}
\end{aligned}
$$

In the exceptional case $p=4$ we have

$$
\left\|b \phi_{n}\right\|_{2} \leq C n^{\frac{2 \alpha}{\beta+2}+\frac{1-\beta}{2(\beta+2)}} \log (n+2)
$$

The following Proposition follows from (7.10), (7.12), (7.13) and Theorem 6 , Our statement of Theorem 6 does not include $\alpha=1$ (and therefore $\beta=2$ ); a proper forumalation and proof of this Theorem for $\alpha=1$ can be found in [1].

Proposition 18. Let $T \in(7.6), b \in L(p, \alpha)$, and define the operator $B$ on $L^{2}(\mathbb{R})$ by $B f=b(x) f(x)$.

Suppose that

$$
\left\{\begin{array}{lll}
\beta-1<p(-4+5 \beta / 2-3 \alpha) & \text { if } & 2 \leq p<4 \\
2>p(3-3 \beta / 2+2 \alpha) \quad \text { if } & 4 \leq p .
\end{array}\right.
$$

Then the system of eigen and associated functions for the operator $T+B$ is an unconditional basis.

Acknowledgements: We would like to thank P. Djakov, K. Shin, A. Shkalikov for interesting discussions related to this work.

\section{REFERENCES}

[1] J. Adduci, B. Mityagin, Eigensystem of an $L^{2}$-perturbed harmonic oscillator is an unconditional basis. arXiv 0912.2722.

[2] E. Akhmerova, Spectral asymptotics for nonsmooth perturbations for the harmonic oscillator. Siberian Mathematical Journal, Vol. 49, No. 6, 968-984, 2008.

[3] T. Kato, Similarity for sequences of projections. Bull. Amer. Math. Soc., 73, 1967, 904-905. 
[4] T. Kato, Perturbation theory for linear operators, Springer Verlag, Berlin, 1980.

[5] M. Naimark, Linear differential operators I, Frederic Ungar Publishing Co., New York, 1967.

[6] F. Olver, Asymptotics and special functions, Academic Press, New York, 1974.

[7] K. Shin, Anharmonic oscillators with infinitely many real eigenvalues and PTsymmetry, SIGMA 6 (2010), 015, 9 pages, ArXiv: 1002.0798

[8] K. Shin, Anharmonic oscillators in the complex plane, PT-symmetry, and real eigenvalues. To appear in Potential analysis. arXiv 1008.0905.

[9] A. Shkalikov, On basisness of root vectors of perturbed self-adjoint operator, Trudy Math. Steklov Institute, 269 (2010), 1-15.

[10] E. Titchmarsh, On the asymptotic distribution of eigenvalues. Quarterly journal of mathematics, 5, 1954, 228-240.

Department of Mathematics, The Ohio State University, 231 West 18th Ave, Columbus, OH 43210, USA

E-mail address: adducij@math.ohio-state.edu

Department of Mathematics, The Ohio State University, 231 West 18th Ave, Columbus, OH 43210, USA

E-mail address: mityagin.1@osu.edu 